\title{
LA FORMACIÓN SUPERIOR EN ARTES MEDIATIAZADA POR DISPOSITIVOS HIPERMEDIALES DINÁMICOS
}

\author{
Federico Buján ${ }^{1}$ \\ Patrícia Silvana San Martín ${ }^{2}$
}

\section{RESUMEN}

El presente artículo aborda aspectos de la formación superior en artes utilizando Tecnologías de la Información y Comunicación (TIC) desde una perspectiva semiótica. Se toma como base el marco teórico y metodológico de los Dispositivos Hipermediales Dinámicos (DHD) en el entendimiento que la complejidad de los fenómenos en cuestión demanda de una visión compleja e interdisciplinaria que permita dar cuenta de las múltiples dimensiones que los componen. Luego de conceptualizar la noción de DHD y sus bases semióticas, se presenta un caso efectuado en el ámbito de una universidad pública de Argentina donde se concretizó, en el marco de un proyecto de investigación, una experiencia de formación superior en artes a partir de la configuración de un DHD contextualizado, en atención a la especificidad disciplinar. Finalmente, se concluye que el enfoque adoptado resulta consistente para la conceptualización de problemáticas de mediatización educativa en el actual contexto físico-virtual.

Palabras clave: Formación Superior en Artes, perspectiva semiótica, Dispositivo Hipermedial Dinámico.

\section{RESUMO}

O presente artigo aborda aspectos da formação superior em artes utilizando Tecnologias da Informação e da Comunicação (TIC) desde uma perspectiva semiótica. Baseia-se no referencial teórico-metodológico dos Dispositivos Hipermediais Dinâmicos (DHD) no entendimento de que a complexidade dos fenômenos em questão demanda de uma visão complexa e interdisciplinar para dar conta das múltiplas dimensões que os compõem. Após conceitualizar a noção de DHD e suas bases semióticas, apresenta-se um caso desenvolvido no âmbito de uma universidade pública da Argentina onde se realizou, no contexto de um projeto de pesquisa, uma experiência de formação superior em artes a partir da configuração de um DHD contextualizado em atenção à especificidade disciplinar. Finalmente, conclui-se que a abordagem adoptada resulta consistente para a conceitualização de problemáticas de mediatização educativa no âmbito do atual contexto físicovirtual.

Palavras chave: Formação Superior em Artes, perspectiva semiótica, Dispositivo Hipermedial Dinâmico.

\section{ABSTRACT}

This article discusses aspects of higher education in arts using Information and Communication Technologies (ICT) from a semiotic perspective. The aspects are based on the theoretical and methodological framework of Dynamic Hypermedia Devices (DHD) on the understanding that the complexity of the phenomena in question demands a complex and interdisciplinary vision able to account for the multiple dimensions that compose them. After conceptualizing the notion of DHD and its semiotic bases, it presents a case that was developed in a public university in Argentina where it was set up, in the sphere of a research project, an experience of higher education in arts through the configuration of a contextualized DHD, in response to the disciplinary specificity. Finally, we conclude that the approach is consistent for conceptualizing mediation education issues in the current physical and virtual context.

Key words: Higher Education in Arts, semiotic perspective, Dynamic Hypermedial Device.

\footnotetext{
${ }^{1}$ Professor e pesquisador no Instituto Universitario Nacional del Arte (IUNA), bolsista pós-doutoral do Consejo Nacional de Investigaciones Científicas y Técnicas (CONICET).

2 Pesquisadora categoria Independente do CONICET, e docente-pesquisadora UNR categoria I (Argentina).
} 


\section{Introducción}

La enseñanza de las artes y, de manera más específica, la formación superior en artes, en el actual contexto físico-virtual, solicita atender tanto a la especificidad disciplinar y de sus objetos de estudio como también a los modos en que son desplegadas las prácticas a partir de la configuración y puesta en obra de nuevos dispositivos técnicos y relacionales sobre la base de redes socio-técnicas educativas. Esta complejidad da cuenta de la singularidad de los procesos de enseñanza y de aprendizaje en dicho dominio específico puestos en relación con las cualidades tanto técnicas como sociales que emergen de las nuevas dinámicas comunicacionales que habilitan los dispositivos en los que se despliegan las prácticas; todo ello, situado en el marco de un contexto institucional determinado.

En este trabajo abordaremos una perspectiva teórica-metodológica en la que se establecen múltiples interacciones entre el campo de la enseñanza de las artes, la semiótica y las mediatizaciones a través de Dispositivos Hipermediales Dinámicos (DHD). Así como la semiótica puede constituir una perspectiva teórica y analítica de gran valor para el estudio de los fenómenos artísticos en los cursos de formación superior en artes, puede también aportar, desde sus marcos conceptuales, entradas analíticas para el estudio de los fenómenos educativos en cuestión, contribuyendo tanto a la teorización como al desarrollo metodológico para la construcción y puesta en obra de redes sociotécnicas colaborativas para el desarrollo de la formación superior en artes.

A tales efectos, presentaremos un caso de desarrollo puesto en obra en el marco del Instituto Universitario Nacional del Arte (IUNA), Buenos Aires, Argentina, en el que a través de un proyecto de Investigación y Desarrollo (I+D), se construyó una propuesta para el desarrollo de la formación superior universitaria en artes a través de redes sociotécnicas colaborativas a partir del marco teórico y metodológico de los DHD -cuyas bases epistemológicas tienen anclaje en una perspectiva semiótica- y que, finalmente, diera lugar al diseño de una carrera de posgrado en el dominio de la crítica y la difusión mediática de las artes.

Consideramos que el mayor desafío no radica tanto en la selección de un sistema informático y en la implementación de determinadas herramientas, aplicaciones y/o servicios, sino más bien en la atención a la especificidad de las prácticas a desarrollar en el entorno virtual, por ende, el análisis de las configuraciones didácticas, la atención del contexto organizacional en que se enmarcan las prácticas y el desarrollo de conocimientos teóricos y metodológicos acerca del funcionamiento y los alcances de los dispositivos habilitados, constituyen ejes centrales para diseñar DHD adecuados a cada caso en 
particular.

Por otra parte, dado el grado de penetración que presentan las TIC en los distintos espacios institucionales, así como también sus alcances para el despliegue de las prácticas en los distintos órdenes de la vida social, se vuelve necesaria su atención e integración en los distintos niveles educativos para promover la inclusión social y, más aún, en el nivel superior, en vistas de desarrollar en los futuros profesionales las habilidades, competencias y disposiciones necesarias para lograr un satisfactorio desempeño profesional acorde con las características que presenta el campo de la producción y para afrontar los diversos desafíos educativos que se presentan en el contexto físico-virtual del siglo XXI en el marco de las múltiples y complejas realidades sociales de nuestra región.

En esta dirección, la formación superior en la crítica y la difusión mediática de las artes (así como también otros dominios de la formación superior universitaria en artes, como por ejemplo, la formación de profesores) requieren de propuestas que promuevan el desarrollo de tales habilidades y disposiciones en los estudiantes, en tanto las mismas constituyen aspectos claves para sus desempeños profesionales futuros (así como también para generar transformaciones de alto impacto social). Es por ello que se torna necesario, en el dominio de esa formación específica (la crítica y la difusión mediática de las artes), el desarrollo de conocimientos y saberes tanto teóricos como prácticos acerca de la producción hipermedial y la circulación discursiva por medio de TIC en ese campo específico de prácticas. Dicha necesidad se torna de capital importancia en ese dominio específico de formación, dadas las condiciones en las que se enmarcan tanto la producción y la circulación de los discursos artísticos en la contemporaneidad y las prácticas de producción de textos críticos y de difusión de las artes en el actual contexto físico-virtual, y en su articulación con el sistema de los medios de comunicación.

Luego de esta introducción y antecediendo al caso mencionado, expondremos los principios teóricos más significativos que conforman el marco teórico-metodológico: por un lado, basados en la teoría de los discursos sociales de Eliseo Verón, presentaremos el núcleo conceptual sobre el que se establece la asimetría estructural de los procesos de producción y reconocimiento en el despliegue de la semiosis y que opera como matriz vincular de los procesos de enseñanza y de aprendizaje. Por otro lado, se conceptualiza la noción de Dispositivo Hipermedial Dinámico (DHD), que operó como matriz de base para el desarrollo de la experiencia. Finalmente se abordarán las conclusiones poniendo en consideración por una parte la necesidad de atender a los lineamientos políticos sobre el Acceso Abierto y, por otra, reafirmando que el campo de la semiótica y de la formación en artes interaccionan en múltiples direcciones. 


\title{
2. Sobre las instancias de Producción y Reconocimiento
}

Los actores sociales se vinculan entre sí a partir de un conjunto de relaciones que dan lugar a múltiples modos de intercambio:

\begin{abstract}
En síntesis: la comunicación humana es necesariamente "mediada" en todos sus niveles, desde lo micro hasta lo macro, simplemente porque el sentido solo puede circular materializado; desde este punto de vista la conversación cara-a-cara entre dos individuos es tan "mediada" como la circulación planetaria de un partido de fútbol. La diferencia crucial es que en la transmisión del partido de fútbol, la semiosis humana está mediatizada y en la conversación no lo está. Ninguna conversación humana es "directa" (por oposición a mediada) pero la mediatización produce (...) importantes alteraciones de escala. (VERÓN, 2013: 148)
\end{abstract}

Focalizando nuestro interés en el actual contexto educativo físico-virtual, y específicamente, en la educación superior, sostenemos la necesidad de indagar acerca de los intercambios discursivos que se establecen en las prácticas de formación. Partiremos de considerar la existencia de dos instancias en toda relación de intercambio discursivo: la de producción y la de reconocimiento. Entre dichas instancias opera la circulación de paquetes de materias significantes (escriturales, verbales, sonoros, visuales, etc.) organizados de forma muy diversa y encabalgados en diferentes modos de enlace entre esas instancias.

Este principio general nos conduce inmediatamente a postular que será necesario para la producción de esta circulación la puesta en obra de gramáticas de producción que organicen la materia significante de cierta manera $y$, en el otro polo, gramáticas de reconocimiento que, al vincularse con esas materias significantes, dan lugar a asignaciones de sentido.

Teniendo en cuenta este principio general podemos señalar que existe entonces una diferencia entre las posiciones de la relación producción-reconocimiento: en producción se pone en obra cierto conjunto de reglas que organizan a esas materias significantes $y$, en el otro extremo (en la instancia de reconocimiento), otro conjuntos de reglas (diversas, por la heterogeneidad de los participantes) que hacen que estas materias significantes, instaladas en una situación socio-tecnológica determinada, den lugar a asignaciones de sentido y a la co-construcción de conocimiento.

Toda producción de sentido, de ese modo, se realiza siempre a partir de una manifestación material y, como acabamos de señalar, supone que se establezca una relación entre las instancias de producción y de reconocimiento. El emplazamiento que da lugar a que se establezca la relación entre dichas instancias, y en las que un conjunto de 
técnicas permiten la circulación de materias significantes, opera y se pone de manifiesto dentro de una situación socio-técnica que permite su instalación social.

En este sentido, las modalidades vinculares que se configuran a través de las prácticas sociales comportan siempre una relación de intercambios materiales y una posición situacional de los actores. De ese modo, las relaciones entre técnicas de producción y técnicas sociales de instalación dan lugar a la configuración de dispositivos relacionales en los cuales los actores están inmersos y que, a la vez, son constituidos por medio de las interacciones que estos últimos establecen.

Del mismo modo, un entorno de formación mediatizado a través de la WEB, supone la configuración de un dispositivo en el que los intercambios discursivos, los desplazamientos enunciativos y los diversos tipos de vínculo que se establecen entre los actores se ponen en obra a través de diversos recursos tecnológicos 0 , más precisamente, en la articulación entre configuraciones textuales y tecnológicas que darán lugar a la producción de sentido a través de una serie de procesos semio-cognitivos. Nos encontramos así ante la emergencia de una entidad socio-tecnológica en la que se ponen en obra cierto tipo de prácticas sociales, las cuales, a su vez, comportan funciones claramente definidas.

La incorporación de tales configuraciones tecnológicas produce asimismo remodelamientos de los vínculos que tienden a producir relaciones que pueden generar efectos de cercanía, con componentes de semejanza a los que pueden darse en los casos de presencialidad física (cara-a-cara), pero no son idénticos y merecen, por lo tanto, un tratamiento diferente.

A los fines de nuestro estudio, proponemos abordar tales fenómenos desde una perspectiva de la discursividad centrándonos en la noción de dispositivo, dando cuenta, de ese modo, de ciertas especificidades de la articulación entre prácticas pedagógicas y formas de la comunicación mediatizada a través de un Dispositivo Hipermedial Dinámico.

Un dispositivo, entonces, estaría compuesto por la integración de dos dimensiones indisociables: una técnica (o conjunto de técnicas constructivas que comportan una materialidad y una configuración particular) y una social (dada por las relaciones y la situación enunciativa en la que se inscriben las prácticas). De ese modo podemos afirmar que un dispositivo es una entidad compleja en la que la enunciación tiene lugar: lugar en que operan los intercambios discursivos y donde se torna posible el emplazamiento social de los discursos.

Un dispositivo es una instancia, un lugar social de interacción y de cooperación que posee sus intenciones, su funcionamiento material y simbólico y, finalmente, sus propios modos de interacción. La economía de 
un dispositivo -su funcionamiento- determinado por las intenciones, se apoya sobre la organización estructurada de medios materiales, tecnológicos, simbólicos y relacionales que modelizan, a partir de sus características propias, los comportamientos y las conductas sociales (afectivas y relacionales), cognitivas, comunicativas de los sujetos. (PERAYA, 1999: 153)

Estos dispositivos se constituyen, así, como espacios de intercambio e interacción en los que se articulan prácticas pedagógicas y formas de la comunicación mediatizada, comportando de ese modo una gran complejidad en ambos dominios; se configuran entonces como entidades complejas que, dado su funcionamiento dinámico, operan como soporte de la circulación discursiva, tornándose así en el lugar en el que se articulan las instancias de producción y reconocimiento en el marco de los proyecto y procesos educativos desarrollados en esos nuevos escenarios.

\section{El Dispositivo Hipermedial Dinámico (DHD)}

Se conceptualiza como DHD a una red sociotécnica conformada por la conjunción de tecnologías y aspectos sociales que posibilita a los sujetos realizar acciones en interacción -responsable- para educar, investigar, gestionar y producir bajo la modalidad de taller físico-virtual (SAN MARTíN, 2008), utilizando la potencialidad comunicacional, transformadora y abierta de las Tecnologías de la Información y Comunicación (TIC).

Desde esta perspectiva, concebimos al DHD como un dispositivo de enunciación mediatizada que permite el despliegue de distintas prácticas sociales a través de la participación en la comunicación. Las mismas se efectivizan a partir de la emergencia de fenómenos comunicacionales que pueden estar orientados a fines diversos, pero que siempre implican la articulación entre configuraciones textuales y tecnológicas poniendo en marcha el despliegue de procesos semióticos.

Los antecedentes de la teoría y metodología sobre los Dispositivos Hipermediales Dinámicos se fueron gestando a lo largo de más de una década en el marco de una serie de proyectos de I+D oficialmente acreditados por organismos nacionales públicos, los cuales han tenido un impacto significativo en ámbitos académicos, investigativos y productivos. Estos proyectos estuvieron vinculados con la integración de las TIC en distintos contextos organizacionales, con especial foco en instituciones educativas del nivel superior universitario.

Tomar en consideración las características de los contextos institucionales / organizacionales en los que se configura un DHD se torna un aspecto fundamental en tanto que dicho contexto constituye el primer factor que opera como haz de condiciones de 
producción de las prácticas que allí se despliegan. En este sentido, las distintas prácticas sociales allí desplegadas están situadas y enmarcadas en un contexto organizacional que opera como primer regulador de la actividad a desarrollar, por lo tanto, los intercambios discursivos que allí se establecen se encuentran regulados, en gran medida, por las condiciones que comporta cada situación en particular.

El desarrollo de procesos educativos a través de un DHD encuentra, de ese modo, múltiples regulaciones correspondientes al contexto organizacional en el que se enmarcan las prácticas; asimismo, la situación particular en la que se despliegan los intercambios discursivos regulará en gran medida los procesos de referenciación que se establecen entre los actores. Se opera así una suerte de fenómeno de reducción de la complejidad, que permite el despliegue de procesos de ajuste intersubjetivo entre los actores participantes de tales prácticas.

Estos procesos de ajuste intersubjetivo son habilitados por el modo interactivo que posibilita el DHD en el marco de un nuevo contexto físico-virtual-interactivocomunicacional. Contexto, este último, en el que los procesos de ajuste intersubjetivo se desarrollan bajo un conjunto de reglas, normas y restricciones vinculadas fuertemente a las posibilidades comunicativas y al accionar transformador del funcionamiento dinámico y el modo interactivo que comporta el DHD.

Conjunto de regulaciones, por lo tanto, que intervienen en la configuración y la gestión de las relaciones vinculares posibilitadas por lo que hemos denominado como Interactividad-DHD:

Interactividad-DHD es un vínculo intersubjetivo responsable mediado por las TIC que conforma una red sociotécnica generadora del intercambio y edición bidireccional y multidireccional de mensajes y objetos en un marco de trabajo colaborativo, abierto, democrático y plural. (GUARNIERI, 2011: 73)

En esta dirección, conceptualizamos y remitimos a la noción de hipermedial, en lugar de lo multimedial, porque consideramos que nos referimos no a una sumatoria de medios (o formatos) sino a una composición integrada por textos digitales diversos en distintos formatos y soportes tecnológicos (paquetes textuales), que toma en cuenta sus posibles convergencias y divergencias como factores morfológicos expresivos, informativos y comunicacionales (SAN MARTíN, 2003), y cuyo funcionamiento dinámico se establece por medio de la Interactividad-DHD que es posibilitada por las cualidades que comporta el dispositivo.

Sobre la base de este principio general debemos considerar las condiciones de desenvolvimiento del fenómeno, en tanto que sobre él reposa la relación entre 
componentes tecnológicos y condiciones relacionales del vínculo. Condiciones, asimismo, que posibilitan la emergencia de nuevos modos de estar presente en el desarrollo de procesos de formación y de producción académica en el marco institucional. Cabe señalar que en el actual contexto físico-virtual, la presencia física convive con la presencia virtual, moduladas, de manera constante, por las dinámicas de participación de los actores.

Nos encontramos, por lo tanto, frente a procesos mediatizados (sincrónicos y asincrónicos) que a partir de la Interactividad-DHD, dan lugar a fenómenos alejados del equilibrio, en una dinámica de cambio y aceleración permanente. Estos procesos son desplegados por una red socio-técnica que se expresa dando cuenta de la multiplicidad de lo hipermedial.

Las posibilidades derivadas del funcionamiento dinámico que comporta el DHD habilitan, asimismo, modos de participación donde las condiciones de producción y de reconocimiento pueden intercambiarse acordes con un espíritu crítico y democrático. En los procesos educativos y bajo la modalidad de taller físico-virtual, estos intercambios posibilitan que el conocimiento y la producción se construya participativamente tensionando con otros. En este sentido, se posibilita un marco consistente para la producción creativa afín a los dominios particulares en cuestión.

Como puede observarse en lo antedicho, el marco teórico y metodológico sobre los DHD reposa sobre una concepción ética que procura promover prácticas inclusivas y democráticas a través del trabajo colaborativo por medio de las TIC, tendientes al reconocimiento de la diversidad y a la producción colectiva e interdisciplinaria de conocimientos.

Entonces, conceptualizamos al DHD no solamente como una red socio-técnica que habilita una multiplicidad de acciones por parte de los actores participantes para el despliegue de las distintas prácticas que allí se desarrollen sino, principalmente, como espacios de producción discursiva que habilitan -en el campo institucional de la educación superior- el desarrollo de procesos de reflexión crítica en un marco de participación responsable, posibilitando de ese modo el despliegue de propuestas didácticas activas y de producción artística, científica y tecnológica que se distancien de los modelos reproductivistas e instrumentales.

En este sentido, el valor de construir un DHD en la universidad pública radica, tanto en lo teórico como en lo metodológico, en la posibilidad de tener en cuenta las $n$ dimensiones que darían lugar a la pluralidad de condiciones y gramáticas de reconocimiento. Estas $n$-dimensiones se expresan en la materialidad física del extenso campo que involucra el cuerpo, las acciones y los objetos situados en un espacio-tiempo habitado, y lo configurado por lo virtual en sus dimensiones de pensamiento, acto, 
discursos y artefactos. A su vez, en el espacio cultural socio-histórico dan cuenta de la diversidad de las condiciones de producción y gramáticas asociadas a dicha producción. Desde esta perspectiva, asumir y sostener la complejidad sistémica abierta, es otorgar un lugar preponderante a la no linealidad de los procesos de co-construcción de conocimiento. De allí que la noción de DHD no se reduce tecnológicamente a los sistemas "e-learning" sino que, como red socio-técnica, sus alcances son mucho más amplios y sus componentes pueden ser de los más variables. La inclusión y desarrollo de los Repositorios Institucionales de Acceso Abierto es un componente clave en la configuración del DHD.

\begin{abstract}
Cuando la textualidad adquiere un cuerpo denso, materialmente identificable y localizable, se plantea la cuestión de su control: donde está, quién tiene acceso, quien puede negociar con sus contenidos y cómo. Así aparece el tercer aspecto fundamental del proceso de mediatización: las condiciones de acceso, es decir, la dimensión de la circulación. Podemos completar, entonces, este primer modelo del fenómeno mediático, cuyos tres aspectos corresponden a las tres dimensiones de la semiosis: la primeridad en su autonomía; la secundariedad, su persistencia en el tiempo, la terceridad, el conjunto de reglas de circulación, que definen las condiciones de acceso al discurso. (VERÓN, 2013: 200)
\end{abstract}

Entonces, considerando las tres dimensiones de la semiosis, la preocupación por la libre circulación de los bienes intelectuales se expresa en la adhesión que el marco teóricometodológico del DHD tiene acerca de las políticas de Acceso Abierto y al desarrollo de Repositorios Institucionales en las universidades públicas ya que en este ámbito específico de formación habilita nuevos horizontes para el desarrollo de los procesos educativos e investigativos, configurando redes socio-técnicas inclusivas de mayores alcances en el marco del actual contexto físico-virtual.

\title{
4. Acerca de la propuesta proyectual en el IUNA
}

Es desde esta perspectiva teórica y metodológica que se formuló una primera propuesta de trabajo que permitiera desarrollar una experiencia innovadora de formación superior en artes a partir de la configuración de un Dispositivo Hipermedial Dinámico, operando dicho DHD como una suerte de laboratorio semiótico que nos permitiera analizar los procesos de interactividad, las operaciones llevadas a efecto tanto en la enseñanza como en el aprendizaje, y las relaciones vinculares que allí se establecían a partir de diferentes recursos comunicacionales y soluciones enunciativas que fueron estabilizando diversas configuraciones socio-técnicas.

La importancia de atender tanto a las modalidades vinculares que pueden 
configurarse en el DHD como a las potenciales formas de Interactividad-DHD radica en que nos permitiría desarrollar procesos formativos adecuados a las necesidades de cada caso en particular, en su especificidad. En este sentido, nos permitiría atender tanto a la diversidad que presentan las propuestas educativas y los objetivos específicos de cada una de ellas así como también a la singularidad y diversidad de los actores que participan en dichos procesos de formación, posibilitando, así, integrar las modalidades de trabajo de los docentes a cargo de los cursos atendiendo a las variantes tanto genéricas como estilísticas que comportan sus prácticas de enseñanza.

Nos referimos, por lo tanto, a la posibilidad de desarrollar redes socio-técnicas flexibles y sensibles al contexto-problema: DHD adecuados a la especificidad de los dominios en cuestión y, de manera particular, a las prácticas formativas que se pretende desarrollar en un contexto institucional determinado. Así, atender las condiciones de funcionamiento del dispositivo y las condiciones de desenvolvimiento de los fenómenos en cuestión nos habilitó plantear un diseño preliminar de DHD acordes a las necesidades de cada caso en particular.

Otro aspecto a ser considerado es el que refiere a los modelos metodológicos a los que se recurre en este tipo de formación (vinculados, en muchos casos, con las características de los sistemas informáticos utilizados y las gramáticas de producción que subyacen a ellos) ya que, en muchos casos, no suelen presentar la flexibilidad necesaria para adecuarse a las características particulares de los programas formativos que se pretende desarrollar. Este aspecto se articula también con el problema de la especificidad de la formación, en tanto que deben considerarse no sólo las cualidades particulares del universo disciplinar sobre el que se trabajará sino también las características particulares y los objetivos específicos de cada uno de los cursos a desarrollar.

Es por ello que es central trabajar en la configuración de modelos interactivos, flexibles y dinámicos adecuados a la especificidad de cada caso en particular en vista de desarrollar procesos formativos satisfactorios $\mathrm{y}$, para ello, es necesario partir de un diagnóstico acerca de las prácticas educativas que atienda a las configuraciones didácticas y a las estrategias de intervención pedagógica a las que se recurre en cada uno de los casos, a las características particulares del tipo de formación, a la naturaleza de los materiales puestos en obra, a los tipos de intercambio que se busca promover entre los actores y a los tipos de vínculo que se establecen en dichos procesos educativos.

En esta dirección, un diagnóstico de tal naturaleza orientaría el diseño y la coconstrucción de Dispositivos Hipermediales Dinámicos adecuados a cada caso en particular. Por otra parte, permitiría no sólo la selección de recursos virtuales interactivoscomunicacionales que podrían resultar adecuados para el despliegue de los procesos 
educativos en cada caso sino también habilitar la posibilidad de desarrollar nuevos recursos para ampliar la flexibilidad del dispositivo y adecuarse así, de manera pertinente, a las características particulares de la formación en cada área del conocimiento y en cada programa de formación.

En este sentido, la relevancia social de esta problemática solicitaba con urgencia el desarrollo de propuestas acordes a las características de dicho dominio. Soluciones, por lo tanto, que no podían quedar reducidas a la mera implementación tecnológica de los denominados sistemas e-learning sino que requerían del desarrollo de conocimientos teóricos y metodológicos acerca de los DHD y de las dinámicas que comportan en el marco de lo académico-formativo.

Tomando en consideración este conjunto de problemáticas es que emprendimos nuestro trabajo. El estudio se enmarcó de manera específica dentro del proyecto de investigación PICT 38120, financiado por la Agencia Nacional de Promoción Científica y Tecnológica (ANPCyT) y radicado en el Área Transdepartamental de Crítica de Artes (ATCA) del Instituto Universitario Nacional del Arte (IUNA); proyecto que tuvo como objetivo general la construcción de un modelo metodológico para el desarrollo de la educación superior en la crítica y difusión de las artes por vía de la aplicación de recursos virtuales interactivos.

Las experiencias llevadas a efecto para dar lugar a los desarrollos experimentales tomaron como unidades de análisis tres unidades curriculares de la carrera de Especialización en Producción de Textos Críticos y de Difusión Mediática de las Artes, dependiente del ATCA del IUNA. Avanzada la investigación se realizaron también transferencias a otros cursos de grado y pregrado universitario tanto al interior de esa unidad académica como así también en el Área Transdepartamental de Formación Docente de la misma universidad (y también a espacios de trabajo de grupos de investigación y de gestión académica).

La carrera de posgrado referida fue creada en el año 2003, dando lugar a una formación específica y con pocos antecedentes (o sólo parciales) tanto en el ámbito nacional como en el exterior. La singularidad de la carrera deviene de la atención prestada a la especificidad cultural de las sociedades actuales y en especial a los modos en que se articulan las artes -y las prácticas de producción estética en general- con los medios de comunicación.

La experiencia en el dictado de dicha carrera, en el transcurso de las primeras cuatro cohortes (anteriores al inicio del proyecto), puso en evidencia el peso de algunas propiedades del campo de desempeño circunscrito, a partir del reconocimiento de que se trata de una enseñanza que, más allá de los aportes técnicos, teóricos o de destrezas, 
posibilita un aprovechamiento que se dirige hacia una modificación del posicionamiento (punto de vista) del futuro profesional, habilitándose así una relación diferente y más productiva con el objeto. Por otra parte, los resultados del dictado de la carrera en esas primeras cohortes habían sido satisfactorios tanto por el porcentaje de egresados (en relación con el número de ingresantes efectivos a la carrera) como por la calidad de los trabajos producidos (según la evaluación de los docentes a cargo de los seminarios y talleres que la componen).

A partir del reconocimiento y del valor asignado al posicionamiento que debían adoptar los futuros profesionales en formación en el marco de la carrera es que se formuló, a modo de hipótesis general y en atención al objeto de la investigación, que las posibilidades de frecuentación y diversificación del acceso a la información, sumadas a la posibilidad de intervenir sobre la textualidad de manera interactiva a partir del desarrollo de procesos colaborativos a través de la modalidad de taller, favorecerían el vínculo requerido para el desarrollo de la práctica crítica y la de difusión de las artes, lugar profesional que difiere tanto del modelo de comunicación característico del productor como del correspondiente al espectador. Tal vínculo constituye la base del posicionamiento en el contexto de la actividad profesional, la que conlleva tanto un acercamiento a la información como a su procesamiento, y que las propuestas resultantes de las experiencias desarrolladas en el marco del proyecto debían favorecer y sistematizar.

El desarrollo de un modelo metodológico, a tales fines, requería de un aporte productivo y evaluativo original que permitiera afrontar, de manera sistemática, los nuevos requerimientos de composición hipermedial de los discursos críticos y de difusión artística, en tanto constituye un aspecto clave para el desempeño profesional del crítico y difusor de las artes en la contemporaneidad. Es en esta dirección que se propuso, en el marco de la investigación, desarrollar e implementar tecnología sensible al contexto-problema considerando tanto las características propias de lo institucional como la diversa y compleja realidad de los actores sociales en los marcos organizacionales.

\section{Las fases de la experiencia}

El diseño de Dispositivos Hipermediales Dinámicos para el desarrollo de procesos educativos en actual contexto físico-virtual requiere, como señalamos, partir de un diagnóstico acerca del contexto en que se enmarcan las prácticas, atendiendo las cualidades específicas que presentan las configuraciones didácticas en cada caso en particular.

El estudio abarcó entonces tanto las problemáticas específicas de los cursos 
seleccionados como también un relevamiento acerca de la producción teórica y metodológica en el campo de la educación mediada por las TIC de los últimos años y las posibilidades que ofrecen los sistemas informáticos contemporáneos diseñados específicamente para el desarrollo de procesos educativos.

Tanto el conjunto de instancias que conformaron dicho recorrido como el conjunto de acciones llevadas a efecto para dar cumplimiento a cada una de ellas tuvieron un horizonte en común: el de generar estrategias de pasaje (una transposición semiótica) de las prácticas de enseñanza que ya habían sido desarrolladas de manera satisfactoria en el marco institucional del ATCA-IUNA en la modalidad con presencialidad plena (TRAVERSA, 2011: 19) a un nuevo escenario plenamente mediatizado a partir de la configuración de un DHD especialmente diseñado en atención a las características singulares de cada uno de los casos involucrados en la experiencia. Como puede observarse, las condiciones y gramáticas de producción fueron tratadas en la fase diagnóstica del proyecto a partir de la experiencia brindada por los docentes involucrados en cada uno de los tres cursos seleccionados.

Es en este sentido que para poder desarrollar e implementar los diversos diseños en cada uno de los Prototipos Experimentales resultaba indispensable examinar el tipo de operaciones que eran llevadas a efecto para el desarrollo de las prácticas de enseñanza (en la modalidad presencial), los modos en que se gestionaba el contacto entre los actores y los tipos de vínculos que se buscaba promover en cada uno de los casos. De allí que fuera necesario partir de un diagnóstico y un posterior análisis sobre ese conjunto de aspectos en vista de precisar las configuraciones didácticas que comportaba cada una de las unidades curriculares que participarían en el desarrollo del modelo experimental. Punto de partida y aspecto diferencial de este estudio que nos permitió precisar las características de las configuraciones didácticas de cada una de esas unidades curriculares, atendiendo a las instancias de producción y reconocimiento.

Para dar cumplimiento a ésto, las tres Unidades de Análisis que se correspondieron con tres unidades curriculares de la carrera de posgrado fueron seleccionadas a partir de un doble criterio:

a. Cada una de ellas parecía comportar características diferenciales tanto en lo que correspondía a la sustancia temática objeto de la enseñanza como a las habilidades y destrezas que buscaban promover para la formación de profesionales en el campo de la crítica y difusión de las artes.

b. Cada una de ellas se desarrollaba en momentos claramente diferenciados de la carrera (fase inicial, fase intermedia y fase terminal), lo cual suponía la posibilidad de efectuar un seguimiento diacrónico de los desarrollos experimentales y 
capitalizar progresivamente los aportes producidos en cada unidad de desarrollo.

Estas Unidades de Análisis (UA), que darían lugar al desarrollo de los Prototipos Experimentales (PE), correspondieron a las siguientes unidades curriculares de la carrera mencionada:

UA1: Seminario "Problemáticas del Arte Contemporáneo" (PAC)

UA2: Seminario "Semiótica de las Artes" (SA)

UA3: "Taller de Producción" (TPr)

Sintéticamente, las experiencias desarrolladas estuvieron integradas por 8 fases: la Fase 1 consistió en la descripción preliminar de las asignaturas previstas y material bibliográfico utilizado en las situaciones presenciales con el propósito de construir un modelo virtual que atendiera a los objetivos del proyecto. La Fase 2 abarcó el Macrodiseño del DHD y la prueba preliminar del modelo virtual. Por otra parte, esta fase incluyó también un profundo trabajo colaborativo y de análisis metacognitivo junto con los docentes participantes de la experiencia a través del modelo experimental en el que focalizaron sobre las operaciones que ponían en obra (muchas veces, de manera naturalizada e inconsciente) en el despliegue de sus prácticas; autorreflexión y análisis sobre sus propias prácticas que se constituyó un aspecto clave para efectuar la transposición de sus prácticas de enseñanza desde un universo a otro. Las Fases 3, 4 y 5 correspondieron al desarrollo e implementación de los Primeros Prototipos Experimentales (PPE) de las unidades curriculares mencionadas: "Problemática del arte contemporáneo", "Semiótica de las artes" y "Taller de producción" en el entorno virtual, recurriendo a grupos de control y llevándose a cabo, posteriormente, un análisis y ordenamiento de los resultados obtenidos (Fase 6). Los espacios de trabajo fueron configurados sobre el Entorno Colaborativo Sakai, y otras herramientas de la WEB 2.0, constituyendo la primera experiencia en el ámbito de la educación superior en Argentina en utilizar esa tecnología. La Fase 7 correspondió a la revisión y ajustes de los PPE tras la discusión de los resultados referidos y al desarrollo e implementación de los Segundos Prototipos Experimentales (SPE) que dieron lugar a la evaluación global de la experiencia. Finalmente, se procedió a la formulación de conclusiones y a la elaboración de un informe final (fase 8) a la par que se desarrollaron un conjunto de transferencias al interior de la institución que aportaron también valiosos insumos para el análisis; elementos claves para observar la variabilidad de la mediatización y el funcionamiento dinámico del DHD así como su adaptabilidad a otras configuraciones didácticas y al tratamiento de otros tópicos en el marco de la formación superior universitaria en artes. 


\section{Hacia una nueva corporeidad}

Las relaciones vinculares que se configuraron en el DHD se encontraron en gran medida condicionadas por las cualidades técnicas que presentaban los recursos tecnológicos a los que se apeló en cada caso para el desarrollo de los procesos educativos. A medida que avanzábamos en el desarrollo de la investigación se volvía cada vez más claro el modo en que cada uno de los recursos tecnológicos incluidos en el DHD intervenía como regulador de la actividad discursiva, condicionando los modos de intervención y la manera de poner en funcionamiento la actividad enunciativa. Por otra parte, se sumaba a la funcionalidad operativa que comportaba cada uno de los recursos tecnológicos la variabilidad en los usos de tales recursos por parte de los docentes, lo cual daba lugar a que se configuraran dispositivos relacionales diferentes aún incluso cuando se apelaba al empleo de las mismas herramientas; de ese modo, se constató la manera en que una misma técnica puede operar de distinto modo según los usos que se hagan de las mismas y según las prácticas sociales en las que se inscriban.

En esa misma dirección, los efectos de cercanía o distancia que se producían entre los actores dependían, al menos en parte, de esas condiciones particulares en las que se inscribían los intercambios discursivos. Pero además, se sumaba a esos condicionantes derivados del funcionamiento operativo de los recursos tecnológicos y de los usos específicos llevados a efecto por los docentes (los cuales se encontraban en consonancia con las configuraciones didácticas estabilizadas en cada uno de los casos), la complejidad que comportaba cada escena enunciativa que allí se desplegaba y, más específicamente, la escenografía (MAINGUENEAU, 2009: 79) que se construía en cada situación de comunicación.

El conjunto de marcadores textuales, deícticos espacio-temporales y modalidades enunciativas (identificables en la superficie discursiva) que intervinieron como moduladores de los enunciados, dieron lugar a la configuración de modos vinculares particulares entre los enunciadores y co-enunciadores que operaron en esos procesos educativos. Pero además, atendiendo a los modos en que los distintos elementos discursivos fueron dando lugar a la conformación de un ethos discursivo (MAINGUENEAU, 2009: 90) en cada una de las intervenciones de los actores, logramos aproximarnos con mayor precisión a las múltiples y variadas tonalidades que allí se desplegaron.

En esta misma dirección, la problemática en torno al cuerpo de los actores cobró aquí un particular interés. Si por un lado es cierto que el cuerpo de los actores no se encuentra presente físicamente en el entorno virtual (aunque su cuerpo participa 
efectivamente al interactuar con las interfaces del entorno a través de sus dispositivos naturales -sus analizadores naturales-, así como también participan sus sistemas de conciencia por medio de los acoplamientos estructurales que posibilitan el establecimiento de relaciones de interpenetración sistémica), a partir del conjunto de elementos discursivos y enunciativos que acabamos de mencionar, los actores construyen/adquieren una suerte de corporeidad en el entorno virtual.

[...] en ese medio en el que uno no cree que va a tener corporeidad, finalmente hay cuerpo [...] el dispositivo construye gestualidad, construye afectividad, construye la personalidad de cada uno. Uno cree que no estando de manera presencial, el otro no se va a enterar de quién es uno. Y la verdad es que se entera igual. Con todos esos paratextos, metatextos, chats, foros, anuncios, cada uno de nosotros me parece que adquirió cuerpo, personalidad, tono y también lo adquirieron los alumnos [profesora a cargo del seminario Semiótica de las Artes, participante del proyecto, entrevistada por Oscar Traversa] (TRAVERSA, 2011: 136).

Es mediante tal corporeidad que se vuelve posible la construcción de afectividad, de gestualidad, de singularidades que se hacen presentes en los intercambios discursivos desplegados en el DHD; elementos, éstos, que operan como moduladores de las relaciones vinculares que allí se establecen.

De esta manera, el sólo hecho de publicar un anuncio en el entorno virtual (ese simple acto de enunciación), involucra una serie de matices y variaciones posibles que se materializan en los enunciados (en tanto configuración de marcadores) y da lugar al despliegue de efectos de sentido (en la instancia de reconocimiento) que también intervienen en la configuración de las relaciones vinculares. Los enunciados, de ese modo, siempre se encuentran modulados (CULIOLI, 2010: 29), produciendo diversas resonancias o inflexiones de sentido (TRAVERSA, 2011: 22).

Esta serie de elementos a los que nos venimos refiriendo da lugar, por una parte, a la construcción de la singularidad de cada uno de los participantes al aportar y materializar su propia complejidad en el entorno y, por otra parte, da lugar a la construcción de una serie de formaciones imaginarias respecto de esos actores intervinientes en la comunicación desplegada en el DHD (formación imaginaria sobre "el/los otro/s" y sobre sí mismo), las cuales también intervienen como condiciones de producción discursiva y operan en la configuración de los vínculos que se establecen entre los actores.

Esa dimensión corpórea que los actores construyen/adquieren en el entorno virtual, permite entonces la manifestación de rasgos estilísticos que los diferencian, que los distinguen, que los singularizan... y, al mismo tiempo, da lugar a efectos de cercanía semejantes -aunque no iguales- a los que pueden derivarse de los intercambios 
establecidos en los vínculos plenos (TRAVERSA, 2011: 19), los cuales involucran una coalescencia tanto temporal como espacial.

Como ejemplo de esa corporeidad de los actores que emerge en la actividad discursiva desplegada en el DHD, incluimos a continuación un fragmento de los intercambios iniciales que se establecieron al momento de dar inicio al primer encuentro de Chat programado en el marco del PPE del seminario Semiótica de las Artes, donde resulta fácil advertir entonaciones y rasgos de emotividad:

Silvana: hola! estoy re ansiosa por la reunión

Paula: hola a todos!

Prof.: hola Paula, Silvana, Alejandra

Silvana: hola [nombra a Prof]

Prof.: Qué momento, no?

Prof.: quieren empezar con alguna pregunta

Paula: uy! qué emoción en vivo y en directo!

Silvana: tengo problemas con la conexión, auxiliooooooooo, me siento un poco inestable

Stella Maris: Hola a todos

Prof. 1: bueno, la inestabilidad es casi un "estilo de época"

Alejandra: Hola a todos!

Prof. 1: Hola Stella

Stella Maris: que tal [nombra a Prof.]

Stella Maris: estamos en tiempo real!

Silvana: perdonnnnnnnn, pero tengo problemas con la página, no lo puedo creeeeeeer!!

Silvana: ahora sí, no se qué toqué pero lo solucioné

La presencia que adquirieron los actores participantes a partir de sus intervenciones en el DHD se desplegó con amplias modulaciones y diferentes resonancias a través de los distintos espacios habilitados en cada uno de los Prototipos Experimentales. Por otra parte, estas condiciones particulares en las que se enmarcó la actividad discursiva desplegada en el DHD evidenciaron, de manera notoria, las diferencias que comportaban entre sí las distintas unidades curriculares que fueron puestas en curso. Del mismo modo, se puso en evidencia la singularidad estilística de cada uno de los docentes en el desarrollo de sus prácticas de enseñanza y en el conjunto de las intervenciones pedagógicas llevadas a efecto.

Estos múltiples procesos de ajuste intersubjetivo a los que nos hemos venido refiriendo, y que dieron lugar a la configuración de distintas relaciones vinculares en cada uno de los casos, fueron habilitados por el modo interactivo que posibilitó el DHD en el marco ese nuevo contexto interactivo-comunicacional y gracias al accionar transformador del funcionamiento dinámico que comporta el DHD. La Interactividad-DHD desarrollada en ese contexto posibilitó, de ese modo, el despliegue subjetivo e intersubjetivo de los actores participantes por medio de los recursos comunicacionales que habilitaron las herramientas 
hipermediales y sobre la base de las cualidades técnicas que comportaron los dispositivos puestos en obra, operando, ambos aspectos, como factores intervinientes en la producción y circulación discursiva y en la configuración de los vínculos en el DHD.

En síntesis: la sumatoria de las condiciones tanto técnicas como sociales que operaron como factores de producción discursiva en el DHD, el gran dinamismo que habilitó la Interactividad-DHD allí desplegada y las múltiples variantes que se fueron produciendo en relación con las configuraciones vinculares establecidas en los distintos espacios habilitados en el entorno virtual, dieron lugar a una gran variabilidad en el desenvolvimiento de los fenómenos en cuestión, mostrando una gran flexibilidad del DHD para adecuarse a las características específicas de cada una de las unidades curriculares y permitiendo el despliegue de la singularidad de los actores a los efectos de establecer el vínculo requerido para ese tipo específico de formación, confirmando, de ese modo, las hipótesis formuladas inicialmente. Así, observando los resultados de los productos, podemos afirmar que la circulación como nivel tres de la semiosis habilitó nuevas condiciones de producción tanto en los dominios específicos como en los procesos de enseñanza y de aprendizaje.

Por otra parte, sobre la base de la concepción semiótica adoptada dentro del marco teórico y metodológico del DHD, este modelo experimental para el desarrollo de la formación superior universitaria en artes a través de la configuración de un DHD operó como plataforma de base para el diseño de una propuesta de mediatización de los procesos educativos en el ámbito del Área Transdepartamental de Crítica de Artes del IUNA, y dio lugar a la creación de una nueva carrera de posgrado (maestría) en el área referida.

\section{Conclusiones}

En el trayecto del presente artículo, hemos abordado algunas problemáticas y desafíos que comporta una propuesta de desarrollo para la formación superior en artes en atención a las cualidades del actual contexto físico-virtual, posicionándonos, a tales efectos, desde una perspectiva semiótica.

Se conceptualizó la noción de Dispositivo Hipermedial Dinámico (DHD) mostrando sus posibles alcances para la puesta en obra de procesos educativos así como sus contribuciones para el desarrollo de procesos investigativos, operando allí la asimetría estructural entre las instancias de producción y reconocimiento como núcleo conceptual de base.

Luego de ello, se sintetizó el modo en que se procedió en un caso específico para 
el desarrollo de una propuesta de formación superior en artes a partir de la configuración de un DHD en el ámbito de una universidad pública Argentina, dando cuenta de las posibilidades que esta perspectiva despliega.

Consideramos, a partir de todo lo expuesto, que aportar propuestas teóricometodológicas para el desarrollo de la investigación y la formación superior universitaria en artes que posibiliten a la comunidad de docentes, investigadores y estudiantes la construcción y participación responsable en redes socio-técnicas colaborativas para educar e investigar, debe estar en consonancia con las actuales políticas de Acceso Abierto (AA) al conocimiento, promoviendo la publicación en Repositorios Digitales Institucionales (RDI) de Acceso Abierto (AA) y la producción y diseminación de Objetos Digitales Educativos (ODE). Cabe mencionar que los ODE producidos se constituyen en una retroalimentación central para la configuración de nuevos DHD. Se torna necesario entonces -constituyendo asimismo un desafío para las instituciones de enseñanza del nivel superior- desarrollar estrategias metodológicas que favorezcan la articulación e interoperabilidad entre sistemas, que optimicen el diseño y acceso a los ODE así como también la articulación de sus metadatos a fin de posibilitar un mejor aprovechamiento de los recursos disponibles en los Repositorios Institucionales y en los entornos colaborativos de aprendizaje que componen los DHD.

Por otra parte, la complejidad de los sistemas que se configuran a partir de tales articulaciones y acoplamientos sistémicos, requieren de estrategias metodológicas que conduzcan a aportar un mayor grado de visibilidad a los procesos de interactividad producidos en el marco de los DHD para su evaluación y optimización.

Estos avances, tanto en materia de políticas públicas como en materia de desarrollo tecnológico, demandan repensar los modelos de formación en la universidad, las maneras de desplegar las prácticas y los modos en que se articulan los órdenes técnicos y sociales en estos nuevos escenarios.

El desarrollo de la educación superior en artes se enfrenta en el siglo XXI a nuevos horizontes y a nuevos desafíos que demandan en prospectiva la construcción de modelos de formación adecuados a su especificidad disciplinar. En esta dirección, el marco teórico y metodológico de los DHD, basado en una perspectiva semiótica, puede colaborar en dicha construcción.

Finalmente, hemos dado cuenta que el campo de la semiótica y de la formación en artes interaccionan en múltiples direcciones: ya no sólo como objeto de enseñanza en uno u otro caso o como vías analíticas en contextos de enseñanza, sino como perspectivas de construcción metodológica que se enriquecen mutuamente en la configuración de redes socio-técnicas colaborativas para educación e investigación en el marco del actual 
contexto físico-virtual.

\section{REFERENCIAS BIBLIOGRÁFICAS}

BUJÁN, F. Interpenetración sistémica y procesos educativos. Semeiosis: Semiótica e Transdiciplinaridade em Revista, São Paulo, № 2, ECA-USP, maio de 2011. Disponível em: $\quad$ http://www.semeiosis.com.br/interpenetracion-sistemica-y-procesos-educativos/. Acesso em: set. 2013.

CULIOLI, A. Escritos. Buenos Aires: Santiago Arcos, 2010.

DEY, A.K.; SALBER, D.; ABOWD, G. A Conceptual Framework and a Toolkit for Supporting the Rapid Prototyping of Context-Aware Applications, anchor article of a special issue on Context-Aware Computing. Human-Computer Interaction (HCl) Journal, Vol. 16 (24), 2001.

GUARNIERI, G. El modo interactivo del Dispositivo Hipermedial Dinámico (Tesis doctoral). Facultad de Humanidades y Artes, Universidad Nacional de Rosario (UNR), 2011.

LITWIN, E. Las configuraciones didácticas. Una nueva agenda para la enseñanza superior. Buenos Aires: Paidós, 1997.

LUHMANN, N. Sistemas Sociales: lineamientos para una teoría general. Barcelona: Anthropos, 1998.

MAINGUENEAU, D. Análisis de textos de comunicación. Buenos Aires: Nueva Visión, 2009.

PERAYA, D. Médiation et médiatisation: le campus virtual. Revue Hermès, No 25, Paris: CNRS Éditions, p. 153-167, 1999.

SAN MARTíN, P. Hipertexto. Seis propuestas para este milenio, Buenos Aires: La Crujía, 2003.

SAN MARTÍN, P. Hacia un dispositivo hipermedial dinámico. Educación e investigación para el campo audiovisual interactivo. Buenos Aires: Universidad Nacional de Quilmes (UNQUI), 2008.

SAN MARTÍN, P. et al El Dispositivo Hipermedial Dinámico Campus Virtual UNR. Secretaría de Tecnologías Educativas y Gestión, Universidad Nacional de Rosario (UNR), 2010.

SAN MARTíN, P.; TRAVERSA, O. (Comp.) El Dispositivo Hipermedial Dinámico Pantallas Críticas. Buenos Aires: Santiago Arcos, 2011.

TRAVERSA, O. Aproximaciones a la noción de dispositivo, Signo y Seña, No 12, Buenos Aires, Revista del Instituto de Lingüística, Universidad de Buenos Aires (UBA), p. 233-247, abril de 2001.

TRAVERSA, O. Dispositivo-Enunciación: en torno a sus modos de articularse. In: SAN MARTÍN, P. y TRAVERSA, O. El Dispositivo Hipermedial Dinámico Pantallas Críticas. Buenos Aires: Santiago Arcos, 2011, Cap. 1, p. 17-29.

VERÓN, E. La semiosis social: fragmentos de una teoría de la discursividad. Barcelona: 
Gedisa, 1987.

VERÓN, E. Fragmentos de un tejido. Barcelona: Gedisa, 2004.

VERÓN, E. Du sujet aux acteurs. La sémiotique ouverte aux interfaces. In: BOUTAUD, J-J; VERÓN, E. Sémiotique Ouverte. Itinéraires sémiotiques en communication. Paris: Lavoisier, Hermès Science, 2007.

VERÓN, E. La semiosis social, 2. Ideas, momentos, interpretantes. Paidós: Buenos Aires, 2013. 